\title{
Genomics and the challenging translation into conservation practice
}

Authors: Affiliations provided at the end of the document

5 Aaron BA Shafer ${ }^{1 *}$, Jochen BW Wolf ${ }^{1 *}$, Paulo C Alves ${ }^{2}$, Linnea Bergström ${ }^{1}$, Michael W Bruford ${ }^{3}$, Ioana Brännström ${ }^{1}$, Guy Colling ${ }^{4}$, Love Dalén ${ }^{5}$, Luc De Meester ${ }^{6}$, Robert Ekblom $^{1}$, Katie D Fawcett ${ }^{7}$, Simone Fior $^{8}$, Mehrdad Hajibabaei $^{9}$, Jason A Hill ${ }^{10}$, A Rus Hoezel ${ }^{11}$, Jacob Hoglund ${ }^{1}$, Evelyn L Jensen ${ }^{12}$, Johannes Krause ${ }^{13}$, Torsten N Kristensen ${ }^{14}$, Michael Krützen $^{15}$, John K McKay ${ }^{16}$, Anita J Norman ${ }^{17}$, Rob Ogden ${ }^{18}$, E Martin Österling ${ }^{19}$, N Joop Ouborg ${ }^{20}$, John Piccolo ${ }^{19}$, Danijela Popovic ${ }^{21}$, Craig R Primmer ${ }^{22}$, Floyd A Reed ${ }^{23}$, Marie Roumet ${ }^{8}$, Jordi Salmona ${ }^{24}$, Tamara Schenekar ${ }^{25}$, Michael

10 K Schwartz ${ }^{26}$, Gernot Segelbacher ${ }^{27}$, Helen Senn ${ }^{18}$, Jens Thaulow ${ }^{28}$, Mia Valtonen ${ }^{29}$, Andrew Veale ${ }^{12}$, Philippine Vergeer $^{30}$, Nagarjun Vijay ${ }^{1}$, Carles Vilà ${ }^{31}$, Matthias Weissensteiner ${ }^{1}$, Lovisa Wennerström ${ }^{10}$, Christopher W Wheat ${ }^{10}$, Piotr Zieliński ${ }^{32}$

* means these authors coordinated writing of the manuscript.

15 Corresponding author: aaron.shafer@ebc.uu.se 


\begin{abstract}
The global loss of biodiversity continues at an alarming rate. Genomic approaches have been suggested as a promising tool for conservation practice, and we discuss how scaling-up to genome-wide inference can benefit traditional conservation genetic approaches and provide qualitatively novel insights. Yet, the generation of genomic data and subsequent analyses and interpretations are still challenging and largely confined to academic research in ecology and evolution. This generates a gap between basic research and applicable solutions for conservation managers faced with multifaceted problems. Before the real-world conservation potential of genomic research can be realized, we suggest that current infrastructures need to be modified, methods must mature, analytical pipelines need to be developed, and successful case studies must be disseminated to practitioners.
\end{abstract}




\section{Conservation biology and genomics}

Like most of the life sciences, conservation biology is being confronted with the challenge of how to integrate the collection and analysis of large-scale genomic data into its toolbox. Conservation biologists pull from a wide array of disciplines in an effort to preserve biodiversity and ecosystem services [1]. Genetic data have helped in this regard by detecting, for example, population substructure, measuring genetic connectivity, and identifying potential risks associated with demographic change and inbreeding [2]. Traditionally, conservation genetics (see Glossary) has relied on a handful of molecular markers ranging from a few allozymes to dozens of microsatellites [3]. But for close to a decade [4], genomics - broadly defined high-throughput sampling of nucleic acids [5] - has been touted as an important advancement to the field, a panacea of sorts for the unresolved conservation problems typically addressed with genetic data $[6,7]$. This transition has led to much promise, but also hyperbole, where concrete empirical examples of genomic data having a conservation impact remain rare.

Under the premise that assisting conservation of the world's biota is its ultimate purpose, the emerging field of conservation genomics must openly and pragmatically discuss its potential contribution towards this goal. While there are prominent examples where genetic approaches have made inroads influencing conservation efforts (e.g., Florida panther augmentation [8,9]) and wildlife enforcement (i.e., detecting illegal harvest [10]), it is not immediately clear that the conservation community and society more broadly have embraced genomics as a useful tool for conservation. Maintaining genetic diversity has largely been an afterthought when it comes to national biodiversity policies [11,12], and attempts to identify areas that might prove to be essential for conserving biological diversity rarely mention genomics (e.g. $[13,14])$. An obvious reason for this disconnect is that many of the pressing conservation issues (e.g., $[15,16])$ simply do not need genomics, but instead need political will.

The traditional use of genetic data in conservation biology has been historically demarcated into two interrelated areas

50 [3]: i) understanding how evolutionary processes such as genetic drift, selection, and migration shape genetic and phenotypic variation of natural populations and determine population structure; and ii) more specifically, describing the effects of low effective population size on genetic variation and population viability. Nested within these are more general conservation issues, such as resolving taxonomic uncertainties, preserving local adaptation, and offsetting inbreeding depression (Table 1). Whether genome scale data can improve inferences within these two areas and better inform conservation initiatives is still not clear. Further, there are a plethora of uncertainties that practitioners need to be aware of, and considerable obstacles that need to be overcome, before genomics can make the transition to applied 
conservation science. Many of the qualitatively novel aspects of genomic analyses, which include monitoring of epigenetic markers [17], environmental DNA approaches to assay species communities [18], and transcriptome assays [19], are still at an exploratory stage and are far from seeing use in real-world conservation issues.

60

In this essay, we focus first on how traditional applications of genetics in conservation can benefit from scaling-up to genome-wide data. In particular, we highlight two key areas that have received attention in the literature: identifying adaptive loci and the increased resolution afforded to genomic tools. Although the attention is warranted, part of the goal of this essay is to highlight the uncertainties associated with their potential application, and suggest how conservation practitioners should best deal with the uncertainties arising from the novel possibilities genomic data offer. We argue both of these areas are still largely an academic pursuit, and compared to conservation genetics, conservation genomics is far from seeing regular application. We discuss why the uptake of genomics in conservation has been, and will likely continue to be, difficult for practitioners and propose solutions to overcome the gap between basic genomics research and conservation practice.

\section{Scaling-up: what can genomics do for conservation genetics?}

From a conservation geneticist's stand point, the genomic approach offers a dramatic increase in the number of variable genetic markers used (generally single nucleotide polymorphisms: SNPs). This advancement should in principle improve the accuracy of estimating diversity and population demographic parameters of conservation relevance [6,20]. Whole genome sequencing, sequencing targeted portions of the genome [21], and direct genotyping of a random subset of the genome (generically referred to as genotyping-by-sequencing or GBS [22]) permits the assaying of genomic diversity at many orders of magnitude above the traditional number of pre-designed genetic markers. These approaches, GBS in particular, bypass the laborious process of marker characterization, primer development, and genotyping required for microsatellites, and can be compared and combined across labs (which is difficult for microsatellite loci).

80 Genomics further opens up the possibility to screen individuals and populations for adaptive loci, which is suggested by some to be the biggest potential contribution of genomics to conservation [5]. While targeting candidate genes (presumed adaptive) or serendipitously finding neutral markers linked to adaptive loci have not been uncommon in the conservation genetics era, given the low level of linkage disequilibrium often seen in natural populations [23] and polygenic nature of many traits [24], screening the entire genome holds considerably more power. Importantly, experimental systems have given us clues as to the signatures adaptive evolution leaves on the genome [25] and the academic interest in using genomics to explore local adaptation in the wild has grown considerably (e.g., [26,27]). 
Conservation genomics is directly impacted as it draws from the methods and models developed in this basic research.

Numerous reviews have covered these advancements in relation to conservation in detail [6,20,28-33], and novel

90 techniques and analyses are still emerging (Box 1). It is fair to say, however, that to date conservation genomics and the broader fields of ecological and evolutionary genomics are far from mature. While much praise and promise has come with adding genomics to the conservationist's toolbox, challenges and uncertainties that do not exist with conservation genetic data have also arisen.

\section{The detection of adaptive loci}

Nearly every review of conservation genomics has discussed the potential of emerging genomic tools to identify and study genetic variation with fitness consequences, which are potentially involved in local adaptation $[6,20,28,33]$. The inclusion of markers that reflect local adaptation would augment the identification of conservation units and improve detection of genomic regions causing inbreeding depression [6,28,34]. Identifying adaptive markers would also be useful for conserving evolutionary processes (e.g., $[35,36]$ ), and genes associated with increased relative fitness could be propagated via human assistance, possibly including purposeful hybridization to maximise adaptability to changing environments $[37,38]$. This facet of conservation genomics clearly has the potential to provide information on the species, population, and individual level that was inaccessible using traditional genetic markers.

105 However, the detection of adaptive loci is challenging and interpretations are often vague. Screening genomes for loci under selection, let alone providing clear evidence for adaptive variation, is still an active area of academic debate and research $[39,40]$. It is becoming increasingly clear that many genes with small effects underlie phenotypic variation of fitness relevance $[41,42]$, which makes their detection difficult. A further challenge is that the loci underlying adaptation need not exhibit clear signals of recent selection [43], and while studies have shown genomes under pervasive selection with many loci linked to adaptive genetic variation [27], others have documented virtually homogenous genomes with only a few regions of presumed fitness significance [26]. Thus while genome-wide data provides access to the adaptive genomic landscape, the signatures of adaptation vary on case-by-case basis and could simply go undetected. In current practice, adaptive loci are often equated with outlier loci showing extreme levels of population differentiation $[39,44]$. Yet, outlier loci often fall within areas of low recombination and are often the result

115 of non-adaptive processes [39]; in such cases, the outlier regions could simply be reflective of demography, background selection in areas containing genomic features (i.e., centromeres), or sequence assembly artefacts. For example, in a 
genomic study on flycatchers (Ficedula spp.) nearly all outlier peaks were likely not the result of selection imposed by the environment, but instead reflected areas of low diversity generated by linked purifying selection [39,45]. Signals, perhaps prematurely associated with local adaptation, could instead be caused by a number of other processes, such as mate choice [26], selfish alleles or background selection acting on a heterogeneous recombination landscape [46-49]. Many genome data sets of wild populations will have neither a genome assembly to assess spatial distribution of putative adaptive variation [50], nor the necessary information on recombination rates to avoid false positive and false negative inferences of local adaptation.

125 Given the multitude of uncertainties surrounding the detection and interpretation of adaptive loci, how can they best be implemented into a conservation framework? In his seminal essay, Michael Soulé [1] discussed the need for conservation biologists to tolerate some degree of uncertainty, and this will be the case for adaptive loci for the foreseeable future. While explicit conservation frameworks incorporating adaptive loci have been proposed [28], empirical examples and case studies of their application are still lacking. Where clear ecotypes exist, diagnostic outlier loci can be a valuable tool for monitoring stocks (e.g., salmon fisheries; [51]) regardless of their adaptive significance. In less clear cut cases the framework of Funk et al. [28], that advocates for a hierarchical approach using a combination of outlier and neutral loci to manage populations is a valid approach. As there is likely to be a trade-off between genetic uniqueness and genomic diversity [52,53], we do not advocate for managing only outlier loci; instead, where putative genetic contributions to adaptive variation can be identified, it should still be standard practice to incorporate additional information in the form of neutral markers or phenotypic data.

This still leaves a broader question unanswered: how much weight should be given to putatively adaptive variation that appears important at present, but holds no information about future adaptive potential? As the proportion of gene sequences with functional annotations grows, the value of individual loci will increase for conservation (e.g., detecting disease susceptibility [54]), and it is conceivable that loci of known function could be managed and propagated under certain conservation scenarios (e.g., disease outbreak in a small population - see also Box 2). Functional annotation can be borrowed from related species [55] and databases (e.g., Gene Ontology Consortium) under the assumption of orthology, though with unclear implications for the organism in question [56]. But caution is warranted here for one reason: using human genetics as the example, even with whole-genome sequencing efforts on large sample sizes, the 145 success in uncovering the genetic basis of rare recessive diseases - and it is those that will be exposed by inbreeding is still very limited [57]. Almost a decade of genomic work on the endangered California condor (Gymnogyps 
californianus) [58] has yet to uncover the genetic basis for chondrodystrophy - a recessive and lethal form of dwarfism. These examples, combined with the above arguments, suggest that we should not expect, or invest a large effort, in detecting single adaptive genes of real consequence for conservation. Even if detected, the overall impact of individual loci on conservation and management will still remain tangential (e.g., through population models and projections [54]) until genotype-phenotype correlations of the focal species can be inferred with a high degree of certainty.

\section{Increased population genomic resolution}

In the realm of population genetics there are clear advantages that come with ample genome-wide data. Notably, genomic data should improve estimates of population demography [44] that are becoming increasingly amenable to model-based inference using Approximate Bayesian Computation or full likelihood models [59,60]. The characterization of processes such as inbreeding will also be more precise $[34,61,62]$ and genomic data can be a useful monitoring tool [63] or reveal subtle population structure that is relevant for stock management (Box 3). Novel analytical approaches using haplotype information [64] or long stretches of homozygous sites [65] to infer population demographic histories have produced particularly exciting results. But similar to adaptive loci, using genomic data to infer demographic parameters is still very much an area of active academic research [66,67]. User friendly software that accommodates genomic data - a key for practical application - is still limited (e.g. [67-69]), and high performance computer clusters are generally required for data storage and analysis. Demographic studies that have explicitly tested the utility of genomic data have shown that essentially whole genome sequencing should be used [60], estimates can be biased [39,70,71], and some parameters simply cannot be estimated [71]. While genomics does offer increased resolution to addressing many of the outstanding conservation genetic problems (Table 1), we must still accept some, perhaps large, degree of uncertainty in the estimates produced.

Here, we propose that conservation practitioners are best served by focussing on broad scale population genetic patterns that might hold relevance to conservation issues of interest. From a practical standpoint the difference between 3 and 5 migrants per generation is not important, but 3 versus 500 is. Explicit formulation of the role genomics can play in achieving conservation priors - meaning a predetermined objective aimed at improving population viability - is a useful model in this regard [5]. If maintaining genetic connectivity were set as a conservation prior for example, differentiating low versus high migration scenarios with genomic data would clearly be informative. We stress, however, that even 175 with more precise estimates the organism's biology still must be taken into account. If there is undetected family or population substructure represented in the genomic data, the estimated demographic parameters, which generally rely 
on coalescent models, will reflect some hierarchical level (i.e. the region or species) instead of the population of interest at its current state. This effect is illustrated in a conservation study on Chinook salmon (Oncorhynchus tshawytsca), where effective population size estimates inferred with genomic data were occasionally higher than the census size [70]. This is a conceptual issue that also influences conservation genetics, but is an important consideration that will impact the utility of the parameters estimated from genome-scale data that might otherwise be overlooked by naively expecting higher accuracy.

\section{The gap between genomics research and conservation application}

185 We are approaching the $\$ 1,000$ genome [72], meaning that generating genomic information has become increasingly accessible even for non-model organisms with large genome sizes [50]. The pursuit of improved resolution and adaptive loci will transform many conservation genetics labs. Additionally, as life science companies dismantle old instrumentation, discontinue software, and support only the new high-throughput instruments, many conservation genetic applications will simply be forced into using genome-wide approaches [5]. At the same time, significant gaps remain in the transfer of fundamental genomic research to end-user conservation application. The scientific and policypractitioner communities operate in largely separate spheres (Figure 2), and we (the authors) feel that introducing genomics into the equation will increase the gap. In the pre-genomics era of microsatellites and Sanger sequencing, individual labs could start with sample collection and proceed all the way to data analysis and application. With nextgeneration sequencing, data generation is predominately outsourced and data cleaning and analysis often requires expert consultation, creating multiple layers that simply do not exist with conservation genetics. Thus, advances in genomic methods may in fact contribute to an increasing gap between research and application without a concerted effort on the part of both scientists and conservation practitioners to build effective bridges. Broadly speaking, these gaps can be described in terms of the knowledge, tools (i.e., user friendly pipelines), finances, and communications needed to link fundamental research with applied science. Funding to the basic and fundamental research institutions is often not compatible with frontline conservation actions, and will only aid in biodiversity conservation if applied research which is often seen as repetitive and incremental from a basic research perspective - is supported (Figure 2).

\section{Bridging the conservation genomics gap}

In our view, it seems most fruitful in the current academic and conservation frameworks to encourage genome scientists 205 to communicate their research to practitioners and stakeholders and apply their techniques to conservation issues where appropriate. We stress that, for the conservation practitioner, it is not important to differentiate genetic from genomic 
methods; rather it is sufficient to convey that we can now address a broader range of questions than before. The uncertainty in the new approaches should be clearly acknowledged. In the long run, it will be crucial to develop a range of laboratory protocols (e.g., [73]) and fail-safe tools that support conservation genomic applications and deliver tangible benefits to conservation managers (Box 3).

However, there is a more systemic problem with the current state of conservation genomics, in that there is little incentive for academic researchers - whom in many ways lead the conceptual debate and the development of genomic tools vital to application - to engage fully in applied conservation. Applied conservation genomics research is generally not reinforced in current funding schemes, and some academic research is branded as conservation (perhaps only as a selling feature for publication), even when it holds little real-world conservation value. Developing a genomic tool or framework that can be implemented by practitioners requires a level of rigor and repetition that is not conducive in the 'publish or perish' climate. Thus, we must rethink how the academic and conservation community funds conservation genomics research. In particular, we envision a research-policy framework analogous to translational medicine, or colloquially "bench-to-bedside", that enables basic research to have an applied impact [74]. Here, the funding is partly driven by designated conservation questions, and application and uptake are the measured currency, not (just) publications (Figure 2). The applied output, evaluated by experts, leads directly into policy and ultimately cycles back to the funding body. Emerging examples, like the UK's research evaluation framework that specifically evaluates impact beyond academia, and Sweden's split governmental funding bodies with one emphasizing a link to stakeholders, should encourage bridging of the gap.

The key for conservation genomics will be for the academic and policy spheres to communicate in an effort to maintain a firm grasp on conceptual advancements (driven by academic research) and on-site conservation needs (recognized by practitioners). We are not so naive as to think that we can restructure the current academic funding and political climate,

230 but recognizing how the current system fails to deliver tangible results is a starting point. Identifying the critical gains from genomic analysis in relation to drivers like conventions (e.g., Convention on International Trade in Endangered Species: CITES) or extinction risks (e.g., International Union for Conservation of Nature (IUCN) Red List) will be vital in this regard. In other words, if the IUCN or CITES request specific information to inform their conservation decisions, can genomics be the tool to provide it? We would be remiss not to note that some progress has been made bridging 235 these barriers. The IUCN recently formed a Conservation Genetics Specialist Group that evaluates such critical gains, and over the last three years the EU Framework 7 Support Action Project 'ConGRESS' [75,76] has engaged over 300 
stakeholders in Europe and canvassed their opinions on the use of genetics in practical conservation. While a need for the application of genetic technologies could be demonstrated to ConGRESS, a clear link to genomics was not yet evident. Thus, conservation genomics still must prove its worth; explicit examples need to be disseminated to the conservation community of realized gains from genomics.

Genomics will not solve many of the current conservation problems simply because the resolution of these problems depends on societal dynamics and political will. Genomics is also not a substitute for biological replication or good experimental design; more sequencing will not provide an answer per se, and the resolution required to address the 245 question at hand (or conservation prior) needs serious consideration. Still, we believe that genomics can play an integral part in resolving conservation issues. With time will come matured methods, supported pipelines, and hopefully an integrated research-to-application framework that will bridge the conservation genomics gap. We encourage both applied and basic genome scientists to apply their work to conservation issues with the hope of alleviating some of the uncertainties discussed in this essay, and ultimately provide clear examples of conservation genomics in action. 


\section{Acknowledgements}

This manuscript arose from the workshop 'Conservation Genomics: Academic exercise or transition with real-world implications?' organized by ABAS and JBWW held in Wik's Castle, Uppsala, Sweden from 18-21 March 2014. The workshop was funded by the ConGenOmics Initiative of the European Science Foundation (Refnr. 5005), the Swedish Research Council (ID: 70720201), and Uppsala University. The workshop program has been included as supplementary material (Appendix S1). All authors vividly contributed to the discussions and participated in drafting the manuscript. The final version of the manuscript benefited from comments by Paul Craze, Paul Hohenlohe, and an anonymous reviewer.

\section{Author affiliations}

1. Uppsala University, Ecology and Genetics, Evolutionary Biology Centre, 75236, Sweden

2. Universidade do Porto, CIBIO/InBIO \& Faculdade de Ciências, 4485-661, Portugal

265 3. Cardiff University, School of Biosciences, CF10 3AX, United Kingdom

4. Musée national d'histoire naturelle Luxembourg, Population Biology, 2160, Luxembourg

5. Swedish Museum of Natural History, Bioinformatics and Genetics, 10405, Sweden

6. KU Leuven - University of Leuven, Aquatic Ecology, Evolution, and Conservation, 3000, Belgium

7. University of Groningen, Behavioural Ecology and Self-organization, 9712, Netherlands

270 8. ETH Zurich, Integrative Biology, 8092, Switzerland

9. University of Guelph, Integrative Biology, N1G 2W1, Canada

10. Stockholm University, Zoology 106 91, Sweden

11. Durham University, Biological and Biomedical Sciences, DH1 3LE United Kingdom

12. University of British Columbia Okanagan, Biology, V1V 1V7, Canada

275 13. University of Tuebingen, Archaeological Sciences, 72070, Germany

14. Aalborg University, Biotechnology, Chemistry and Environmental Engineering, 9220, Denmark

15. University of Zurich, Anthropological Institute \& Museum, 8057, Switzerland

16. Colorado State University, Bioagricultural Sciences and Pest Management, 80523-1177, United States

17. Swedish University of Agricultural Sciences, Wildlife, Fish and Environmental Studies, 90183 Sweden

280 18. Royal Zoological Society of Scotland, WildGenes Laboratory, EH12 6TS, United Kingdom

19. Karlstad University, Biology, 65188 , Sweden

20. Radboud University Nijmegan, Experimental Plant Ecology, 6500GL, Netherlands 
21. University of Warsaw, Centre of New Technologies, 00-681, Poland

22. University of Turku, Biology, 20014, Finland

285 23. University of Hawai'i at Manoa, Biology, 96822, United States

24. Instituto Gulbenkian de Ciência, Population and conservation genetics group, 2780-156, Portugal

25. Karl-Franzens University Graz, Zoology, 8010, Austria

26. USDA Forest Service, Rocky Mountain Research Station, 59801, United States

27. University Freiburg, Wildlife Ecology and Management, 79106, Germany

290 28. Norwegian Institute for Water Research, Freshwater Biology, N-0349, Norway

29. University of Eastern Finland, Biology, 80101, Finland

30. Wageningen University, Nature Conservation and Plant Ecology, 6708 PB, Netherlands

31. Estación Biológica de Doñana, Conservation and Evolutionary Genetics Group, 41092, Spain

32. Jagiellonian University, Institute of Environmental Sciences, 30-387, Poland 
Table 1. Main areas traditionally addressed by conservation genetics [3], current status of genetic and genomic approaches and the contribution genomics can potentially make. The far-right column details steps required before genomic research can regularly be applied to conservation issues.

\begin{tabular}{|c|c|c|c|}
\hline Category & $\begin{array}{c}\begin{array}{c}\text { Status of conservation } \\
\text { genetics }\end{array} \\
\end{array}$ & $\begin{array}{l}\text { Possible contribution of } \\
\text { conservation genomics }\end{array}$ & $\begin{array}{l}\text { Required for transition } \\
\text { from basic to applied }\end{array}$ \\
\hline \multicolumn{4}{|c|}{ Evolutionary genetics of natural populations } \\
\hline $\begin{array}{l}\text { Demographic inference - } \\
\text { population history }\end{array}$ & $\begin{array}{l}\text { Regularly used } \\
\text { Moderate resolution }\end{array}$ & $\begin{array}{l}\text { Improved accuracy and precision } \\
\text { Finer-scale population structure } \\
\text { Less limited by sample size }\end{array}$ & $\begin{array}{l}\text { Clear understanding of biases } \\
\text { User-friendly software }\end{array}$ \\
\hline Adaptive genetic variation & $\begin{array}{l}\text { Minimally used } \\
\text { Limited to population correlations } \\
\text { [77] or candidate gene approaches }\end{array}$ & $\begin{array}{l}\text { Improved detection of adaptive loci } \\
\text { Management frameworks proposed [28] } \\
\text { Methods still emerging and } \\
\text { interpretations unclear }\end{array}$ & $\begin{array}{l}\text { In-depth validation studies } \\
\text { Genome annotation }\end{array}$ \\
\hline $\begin{array}{l}\text { Quantitative genetic } \\
\text { variation }\end{array}$ & $\begin{array}{l}\text { Limited resolution } \\
\text { Often dependent on pedigrees or } \\
\text { targeted gene approaches }\end{array}$ & $\begin{array}{l}\text { Improved detection of quantitative trait } \\
\text { loci } \\
\text { Active application (e.g., genome-wide } \\
\text { association studies) }\end{array}$ & $\begin{array}{l}\text { Ecological studies } \\
\text { Genome annotation } \\
\text { User-friendly pipelines }\end{array}$ \\
\hline $\begin{array}{l}\text { Taxonomic identification } \\
\text { and general diagnostics }\end{array}$ & $\begin{array}{l}\text { Regularly used } \\
\text { Moderate resolution } \\
\text { Restricted to single individuals }\end{array}$ & $\begin{array}{l}\text { Assay species simultaneously [78] } \\
\text { Improved hybridization detection } \\
\text { Improved detection of pathogens }\end{array}$ & $\begin{array}{l}\text { Defined pipelines (Box 3) } \\
\text { Repeatability }\end{array}$ \\
\hline \multicolumn{4}{|c|}{ ii) Effects of small population size } \\
\hline Inbreeding detection & $\begin{array}{l}\text { Regularly used } \\
\text { Limited resolution [34] }\end{array}$ & $\begin{array}{l}\text { Improved estimates of inbreeding [34,62] } \\
\text { Novel genomic metrics [79] } \\
\text { Assess impact on specific genomic } \\
\text { regions or adaptive loci }\end{array}$ & $\begin{array}{l}\text { User-friendly bioinformatics } \\
\text { Genome annotation } \\
\text { Practitioner demand }\end{array}$ \\
\hline Population viability & Minimally used [80] & $\begin{array}{l}\text { Improved estimates of inbreeding metrics } \\
\text { used in viability models [80] }\end{array}$ & Practitioner demand \\
\hline \multicolumn{4}{|c|}{ Additional applications } \\
\hline Genetic monitoring & Minimally used [11] & $\begin{array}{l}\text { Improved sampling regimes [63] } \\
\text { More powerful biodiversity surveys }\end{array}$ & $\begin{array}{l}\text { Practitioner demand } \\
\text { Government compliance [11] }\end{array}$ \\
\hline Population census & Regularly used & Limited need for application & Practitioner demand \\
\hline $\begin{array}{l}\text { Maternity, paternity, and } \\
\text { kinship analysis }\end{array}$ & Regularly used & $\begin{array}{l}\text { Useful when microsatellite power is } \\
\text { limited [81] }\end{array}$ & Practitioner demand \\
\hline
\end{tabular}




\section{Glossary Box}

305 Adaptive locus: a region of the genome under selection that codes for (or is closely linked to a causative locus) a phenotype with fitness consequences in a particular environment.

Annotation: the process of delineating and assigning function to genetic sequences.

310 Background selection: the loss of genetic diversity at neutrally evolving sites that are linked to sites under purifying selection.

Candidate genes: genes putatively underlying variation in a certain phenotype.

315 Coalescent theory: a retrospective population genetic framework that traces genetic variants of a locus to the most recent common ancestor. Used to infer demographic parameters of population histories.

Conservation genetics: uses genetic markers to conserve biodiversity and help manage species or populations. Traditional genetic markers include: allozymes, microsatellites, and targeted gene sequence.

Conservation genomics: uses genome-wide information to conserve biodiversity and help manage species or populations. Genomic data is derived from high-throughput sequencing technology. Relevant examples are whole genome re-sequencing and targeted approaches like exome sequencing, genotyping-by-sequencing, SNP genotyping, and transcriptome sequencing.

Effective population size: a population genetic convention describing the number of breeding individuals in an ideal population that would lose genetic variation at the same rate as the observed population.

Environmental DNA: is DNA found in environmental samples (e.g., water, soil) that can be used in genetic or genomic analysis. This contrasts traditional approaches that target a specific organism or tissue.

Genetic drift: the loss of genetic variants due to random sampling from one generation to the next.

Genome assembly: the process of ordering and orienting sequencing into a contiguous consensus sequence of the genome.

Genotyping-by-sequencing: the sequencing of a repeatable subset of the genome seeded by restriction enzyme recognition sites. Restriction-site Associated DNA sequencing or RAD-seq is another commonly used term.

340 Haplotypes: a particular combination of alleles at collinear positions along a stretch of DNA.

Inbreeding: the increase of genomic segments in identity-by-descent due to mating between closely related individuals. Results in an increase in homozygosity potentially revealing detrimental recessive alleles with negative fitness consequences.

Linkage disequilibrium: the non-random association of alleles at two or more loci.

Outlier locus: a region of the genome that, based on user-defined criteria (often extreme population differentiation), deviates from the rest of the entire genome.

Orthology: homologous DNA sequence descended from a shared common ancestor

Recombination: the process of genetic exchange between homologous chromosomes, often resulting in a new combination of alleles.

Transcriptome: set of all RNA molecules transcribed from a DNA template. 
360 Figure 1 (in Box 2). Genetic rescue on a population of (a) Bighorn sheep (Ovis canadensis) at National Bison Range, Montana, USA. Two separate introductions successfully increased genetic diversity and reversed the negative effects of inbreeding [82]. Miller et al. [83] used population monitoring and genome-scale data to document the demographic impact of the introductions. (b) The hybrid index based on genome-wide data showed that migrant alleles (from translocated individuals) increased over time, and life history traits including (c) longevity and (d) number of offspring both increased with migrant ancestry.

Figure 2. Schematic for the translation of conservation genomics research to conservation policy and action. Currently there are two largely separate spheres of applied and basic research. The black lines are the basic research to applied workflow with the green lines reflecting the extent to which conservation genetics and genomics currently fit into this scheme - note that conservation genetics has integrated into the applied sphere. The dashed green line represents the gap we see between the academic groundwork readily embracing genomic technology and on-site conservation needs. The grey lines are reflective of the larger framework we envision (and that is emerging) where conservation questions directly fund conservation genomic research and feed into management and biodiversity policies. Importantly, the applied component maintains a firm link to the conceptual advancements driven by basic research. 


\section{Box 1 - An emerging area stemming from ancient DNA technology}

Genomic analysis of ancient samples - considered anything from a museum specimen to archaeological specimens [84] - can establish baseline levels of genetic parameters in ancestral populations prior to demographic declines [85]. This concerns both the amount of genetic variation that might have been lost over time and the origin of contemporary population structure. The timing of population fragmentation and how these are related to past changes in the environment (e.g., anthropogenic impact or climate change) can provide valuable insight into current processes influencing population viability. For example, whether low effective size or high population structure is inherent to the biology of a species or a direct result of recent anthropogenic disturbance is useful information to have when factoring genomic data into conservation planning.

Ancient DNA and genomic approaches provide another advantage in that they are less constrained by sample quality. The latter is illustrated by the fact that entire genomes have been reconstructed, using optimized genomic approaches, from 400,000 year old samples of early hominids [86]. Sample quality is of direct concern to conservation biology, since sample collection and quality become increasingly difficult when the species of interest is rare or elusive in the wild. Conservation biologists often need to resort to museum samples or non-invasive sampling (e.g. scat, hair, scales) of poor quality. Utilizing genomic techniques often employed for ancient DNA studies (e.g., [85,87]), holds potential not only to recover genetic information from the species of interest, but additionally reveal aspects of pathogens (e.g., [88]) that might prove relevant for conservation and management. 


\section{Box 2 Conservation genomics and translocations}

Endangered species are often vulnerable to pathogen outbreaks both in captive breeding populations and in small, isolated natural populations. With high throughput sequencing approaches even trace amounts of pathogen or parasite DNA can be detected from various types of samples (e.g., environmental DNA [18]) and host species can be readily identified [89]. This technology provides a rapid and cost efficient way to identify and monitor pathogen load in populations at risk. Translocation and re-introduction programs could benefit from rapid genomic testing of pathogen load prior to release and select individuals based on desired criteria. Genomic screening could also allow for the selection of individuals according to specific genetic signatures or simply maximum variation. For the purpose of conservation interventions such as the founding of captive populations, performing genetic rescues (i.e., restoring positive growth [90]), or assisting migration, these data could be vital and the ability to monitor the outcomes after such

405 interventions would be enhanced (e.g., Figure 1; [83]). 


\section{Box 3 - From the lab to law: FishPopTrace}

The step change afforded by genomic techniques and the transition from fundamental research to application can both be demonstrated by the development of genomic tools for fisheries management in Europe. The FishPopTrace project funded by the European Union Framework Program 7 utilized genome-wide technologies to identify and genotype SNP

410 markers in four species of commercial fish: cod, herring, hake and sole. These data were used by fishery geneticists to describe populations within species and subsequently to identify minimum SNP panels of maximum power to trace the geographic origin of fish in trade. FishPopTrace revealed population structure at a geographic scale not previously recognized, leading directly to tools for applied conservation (e.g., identification of illegal trade and mislabelling). The UK government and the Marine Stewardship Council are now using the stock traceability information in a regulatory

415 and authenticity verification framework. The challenge for applied conservation labs will be to develop similar pipelines and apply this level of rigour to less charismatic or economically valued species. 


\section{References}

1 Soulé, M.E. (1985) What Is Conservation Biology? BioScience 35, 727-734

2 Frankham, R. (1995) Conservation genetics. Annu. Rev. Genet. 29, 305-327

Frankham, R. et al. (2001) Introduction to Conservation Genetics, Cambridge University Press.

4 DeSalle, R. and Amato, G. (2004) The expansion of conservation genetics. Nat. Rev. Genetics 5, 702-712

5 McMahon, B.J.et al. (2014) How and why should we implement genomics into conservation? Evol. Appl. DOI: 10.1111/eva.12193

6 Allendorf, F.W.et al. (2010) Genomics and the future of conservation genetics. Nat. Rev. Genetics 11, 697-709

7 Frankham, R. (2010) Where are we in conservation genetics and where do we need to go? Conserv. Genet. 11, 661-663

8 U.S. Fish and Wildlife Service (2008) Florida panther recovery Plan (Puma concolor coryi), Third Revision, U.S. Fish and Wildlife Service.

9 Seal, U.S. and Lacy, R.C. (1994) A plan for genetic restoration and management of the Florida panther (Felis concolor coryi), IUCN.

10 Manel, S. et al. (2002) Detecting wildlife poaching: identifying the origin of individuals with Bayesian assignment tests and multilocus genotypes. Conserv. Biol. 16, 650-659

11 Laikre, L. (2010) Genetic diversity is overlooked in international conservation policy implementation. Conserv. Genet. 11, 349-354

43512 Laikre, L. et al. (2010) Neglect of genetic diversity in implementation of the convention on biological diversity. Conserv. Biol. 24, 86-88

13 Sutherland, W.J. et al. (2010) A horizon scan of global conservation issues for 2010. Trends Ecol. Evol. 25, 1-7

14 Carroll, S.P. et al. (2014) Applying evolutionary biology to address global challenges. Science DOI: $10.1126 /$ science. 1245993

44015 Ripple, W.J. et al. (2014) Status and ecological effects of the world's largest carnivores. Science 343, 12414841241484

16 Fyumagwa, R. et al. (2013) Roads as a threat to the Serengeti ecosystem. Conserv. Biol. 27, 1122-1125

17 Danchin, É.et al. (2011) Beyond DNA: integrating inclusive inheritance into an extended theory of evolution. Nat. Rev. Genetics 12, 475-486

44518 Taberlet, P.et al. (2012) Environmental DNA.Mol.Ecol. 21, 1789-1793

19 Vandersteen Tymchuk, W. et al. (2010) Conservation genomics of Atlantic salmon: variation in gene expression between and within regions of the Bay of Fundy. Mol. Ecol. 19, 1842-1859

20 Harrisson, K.A. et al. (2014) Using genomics to characterize evolutionary potential for conservation of wild populations. Evol. Appl. DOI: 10.1111/eva.12149

45021 Mamanova, L. et al. (2010) Target-enrichment strategies for next-generation sequencing. Nat. Methods 7, 111118

22 Narum, S.R. et al. (2013) Genotyping-by-sequencing in ecological and conservation genomics. Mol. Ecol. 22, 2841-2847

23 Poelstra, J.W. et al. (2013) An extensive candidate gene approach to speciation: diversity, divergence and linkage disequilibrium in candidate pigmentation genes across the European crow hybrid zone. Heredity 111, 467-473

24 Robinson, M.R.et al. (2014) Explaining additional genetic variation in complex traits. Trends Genet. 30, 124-132

25 Burke, M.K. (2012) How does adaptation sweep through the genome? Insights from long-term selection experiments. Proc. Roy. Soc. B 279, 5029-5038

26 Poelstra, J.W. et al. (2014) The genomic landscape underlying phenotypic integrity in the face of gene flow in

27 Soria-Carrasco, V. et al. (2014) Stick insect genomes reveal natural selection's role in parallel speciation. Science $344,738-742$

28 Funk, W.C. et al. (2012) Harnessing genomics for delineating conservation units. Trends Ecol. Evol. 27, 489-496

29 Steiner, C.C. et al. (2013) Conservation genomics of threatened animal species. Annu. Rev. Anim. Biosci. 1, 261281

30 Kohn, M.H. et al. (2006) Genomics and conservation genetics. Trends Ecol. Evol. 21, 629-637

31 Angeloni, F. et al. (2011) Genomic toolboxes for conservation biologists. Evol. Appl. 5, 130-143

32 Primmer, C.R. (2009) From conservation genetics to conservation genomics. Ann. N. Y. Acad. Sci. 1162, 357-368

33 Ouborg, N.J. et al. (2010) Conservation genetics in transition to conservation genomics. Trends Genet. 26, 177187

34 Miller, J.M. and Coltman, D.W. (2014) Assessment of identity disequilibrium and its relation to empirical heterozygosity fitness correlations: a meta-analysis. Mol. Ecol. 23, 1899-1909

35 Crandall, K. et al. (2000) Considering evolutionary processes in conservation biology. Trends Ecol. Evol. 15, 290-295

47536 Hoffmann, A.A. and Willi, Y. (2008) Detecting genetic responses to environmental change. Nat. Rev. Genetics 9 , $421-432$ 
37 Barton, N.H. (2008) The role of hybridization in evolution. Mol. Ecol. 10, 551-568

38 Nolte, A.W. and Tautz, D. (2010) Understanding the onset of hybrid speciation. Trends Genet. 26, 54-58

39 Cruickshank, T.E. and Hahn, M.W. (2014) Reanalysis suggests that genomic islands of speciation are due to reduced diversity, not reduced gene flow. Mol. Ecol. 23, 3133-3157

40 Vitti, J.J. et al. (2013) Detecting natural selection in genomic data. Annu. Rev. Genet. 47, 97-120

41 Santure, A.W. et al. (2013) Genomic dissection of variation in clutch size and egg mass in a wild great tit ( Parus major) population. Mol. Ecol. 22, 3949-3962

42 Robinson, M.R. et al. (2013) Partitioning of genetic variation across the genome using multimarker methods in a wild bird population. Mol. Ecol. 22, 3963-3980

43 Ågren, J. et al. (2013) Genetic mapping of adaptation reveals fitness tradeoffs in Arabidopsis thaliana. Proc. Natl. Acad.Sci.U.S.A. 110, 21077-21082

44 Luikart, G. et al. (2003) The power and promise of population genomics: from genotyping to genome typing. Nat. Rev. Genetics 4, 981-994

45 Ellegren, H. et al. (2012) The genomic landscape of species divergence in Ficedula flycatchers. Nature 491, 756760

46 Sandler, L. and Novitski, E. (1957) Meiotic drive as an evolutionary force. Am. Nat. XCI, 105-110

47 Charlesworth, B. et al. (1997) The effects of local selection, balanced polymorphism and background selection on equilibrium patterns of genetic diversity in subdivided populations. Genet. Res. 70, 155-174

49548 Hu, X.-S. and He, F. (2005) Background selection and population differentiation. J. Theor. Biol. 235, 207-219

49 Stephan, W. (2010) Genetic hitchhiking versus background selection: the controversy and its implications. Phil. Trans. R. Soc. B. 365, 1245-1253

50 Ekblom, R. and Wolf, J.B.W. (2014) A field guide to whole genome sequencing, assembly and annotation. Evol. Appl. DOI: $10.1111 /$ eva.12178

50051 Russello, M.A. et al. (2011) Detection of outlier loci and their utility for fisheries management. Evol. Appl. 5, 3952

52 Coleman, R.A. et al. (2013) Balancing genetic uniqueness and genetic variation in determining conservation and translocation strategies: a comprehensive case study of threatened dwarf galaxias, Galaxiella pusilla(Mack) (Pisces: Galaxiidae). Mol. Ecol. 22, 1820-1835

50553 Bonin, A. et al. (2007) Population adaptive index: a new method to help measure intraspecific genetic diversity and prioritize populations for conservation. Conserv. Biol. 21,697-708

54 Robinson, S.J. et al. (2012) Emerging prion disease drives host selection in a wildlife population. Ecol Appl 22, 1050-1059

55 Primmer, C.R. et al. (2013) Annotated genes and nonannotated genomes: cross-species use of Gene Ontology in ecology and evolution research. Mol.Ecol.22, 3216-3241

56 Pavlidis, P. et al. (2012) A critical assessment of storytelling: gene ontology categories and the importance of validating genomic scans. Mol. Biol. Evol. 29, 3237-3248

57 Cirulli, E.T. and Goldstein, D.B. (2010) Uncovering the roles of rare variants in common disease through wholegenome sequencing. Nat. Rev. Genetics 11, 415-425

51558 Romanov, M.N. et al. (2006) Construction of a California condor BAC library and first-generation chickencondor comparative physical map as an endangered species conservation genomics resource. Genomics $88,711-$ 718

59 Gutenkunst, R.N. et al. (2009) Inferring the joint demographic history of multiple populations from multidimensional snp frequency data. PLoS Genet 5, e1000695

60 Robinson, J.D. et al. (2014) ABC inference of multi-population divergence with admixture from unphased population genomic data. Mol. Ecol. 23, 4458-4471

61 Hoffman, J.I. et al. (2014) High-throughput sequencing reveals inbreeding depression in a natural population. Proc. Natl. Acad. Sci. U.S.A. 111, 3775-3780

62 Kardos, M. et al. (2013) Evaluating the role of inbreeding depression in heterozygosity-fitness correlations: how useful are tests for identity disequilibrium? Mol. Ecol. Res. 14, 519-530

63 Hoban, S.et al. (2014) Comparative evaluation of potential indicators and temporal sampling protocols for monitoring genetic erosion. Evol. Appl. DOI: 10.1111/eva.12197

64 Harris, K. and Nielsen, R. (2013) Inferring Demographic History from a Spectrum of Shared Haplotype Lengths. PLoS Genet 9, e1003521

53065 Li, H. and Durbin, R. (2011) Inference of human population history from individual whole-genome sequences. Nature 475, 493-496

66 Excoffier, L. et al. (2013) Robust demographic inference from genomic and SNP data. PLoS Genet 9, e1003905

67 Pinho, C. and Hey, J. (2010) Divergence with gene flow: models and data. Annu. Rev. Ecol. Evol. Syst. 41, 215230

53568 Excoffier, L. et al. (2005) Bayesian analysis of an admixture model with mutations and arbitrarily linked markers. Genetics 169, 1727-1738 
69 Cornuet, J.M. et al. (2014) DIYABC v2.0: a software to make approximate Bayesian computation inferences about population history using single nucleotide polymorphism, DNA sequence and microsatellite data. Bioinformatics 30, 1187-1189

54070 Larson, W.A. et al. (2014) Genotyping by sequencing resolves shallow population structure to inform conservation of Chinook salmon (Oncorhynchus tshawytscha). Evol. Appl. DOI: 10.1111/eva.12128

71 Shafer, A.B.A. et al. Demographic inferences using short-read genomic data in an Approximate Bayesian Computation framework: in silico evaluation of power, biases, and a proof of concept in Atlantic walrus. Under review (included in supplementary material)

54572 Hayden, E.C. (2014) Technology: The \$1,000 genome. Nature 507, 294-295

73 Zieliński, P. et al. (2014) Development, validation and high-throughput analysis of sequence markers in nonmodel species. Mol. Ecol. Res. 14, 352-360

74 Woolf, S.H. (2008) The meaning of translational research and why it matters. JAMA 299, 211-213

75 Hoban, S.M. et al. (2013) Bringing genetic diversity to the forefront of conservation policy and management. Conserv. Genet. Resour. 5, 593-598

76 Hoban, S. et al. (2013) Conservation genetic resources for effective species survival (congress): bridging the divide between conservation research and practice. J. Nat. Conserv. 21, 433-437

77 Shafer, A.B.A. and Wolf, J.B.W. (2013) Widespread evidence for incipient ecological speciation: a meta-analysis of isolation-by-ecology. Ecology Letters

55578 Taberlet, P. et al. (2012) Towards next-generation biodiversity assessment using DNA metabarcoding. Mol. Ecol. 21, 2045-2050

79 Polašek, O. et al. (2010) Comparative assessment of methods for estimating individual genome-wide homozygosity-by-descent from human genomic data. BMC Genomics 11, 139

80 Frankham, R. et al. (2014) Genetics in conservation management: Revised recommendations for the 50/500 rules,

81 Tokarska, M. et al. (2009) Effectiveness of microsatellite and SNP markers for parentage and identity analysis in species with low genetic diversity: the case of European bison. Heredity 103, 326-332

82 Hogg, J.T. et al. (2006) Genetic rescue of an insular population of large mammals. Proc. Roy. Soc. B 273, 14911499

56583 Miller, J.M. et al. (2012) Genomic consequences of genetic rescue in an insular population of bighorn sheep (Ovis canadensis). Mol. Ecol.21, 1583-1596

84 Pääbo, S. et al. (2004) Genetic analyses from ancient dna. Annu. Rev. Genet. 38, 645-679

85 Bi, K. et al. (2013) Unlocking the vault: next-generation museum population genomics. Mol. Ecol. 22, 6018-6032

86 Meyer, M. et al. (2013) A mitochondrial genome sequence of a hominin from Sima de los Huesos. Nature 505, 403-406

87 Perry, G.H. et al. (2010) Genomic-scale capture and sequencing of endogenous DNA from feces. Mol. Ecol. 19, 5332-5344

88 Bos, K.I. et al. (2014) Pre-Columbian mycobacterial genomes reveal seals as a source of New World human tuberculosis. Nature DOI: 10.1038/nature13591

57589 Thiemann, T.C. et al. (2011) Development of a high-throughput microsphere-based molecular assay to identify 15 common bloodmeal hosts of Culex mosquitoes. Mol. Ecol. Res. 12, 238-246

90 Carlson, S.M. et al. (2014) Evolutionary rescue in a changing world. Trends Ecol. Evol. 29, 521-530 
Figure 1 in Box 2

(a)

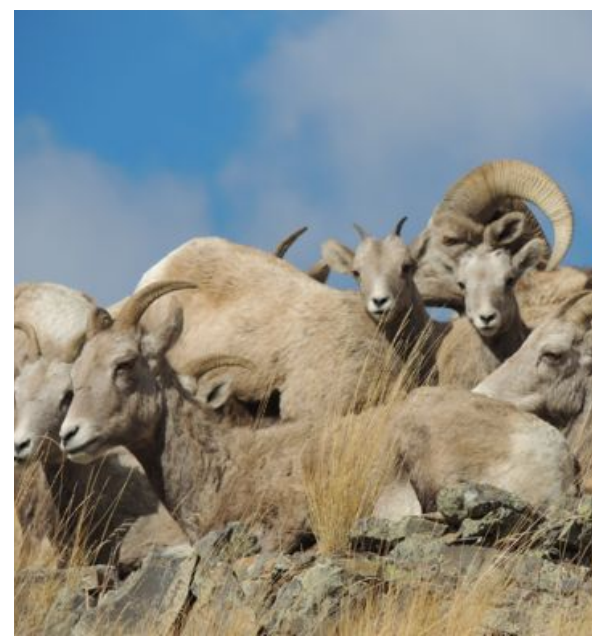

(c)

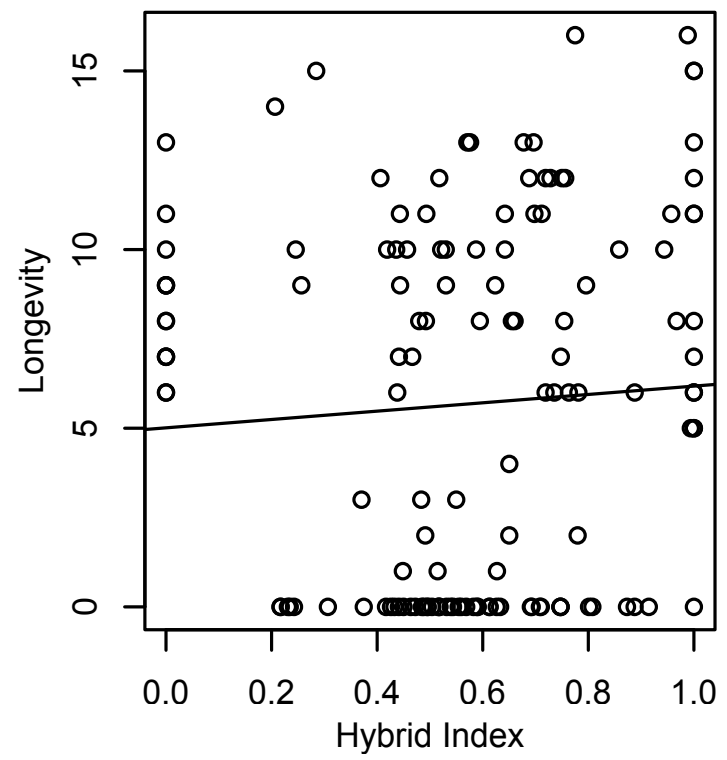

(b)

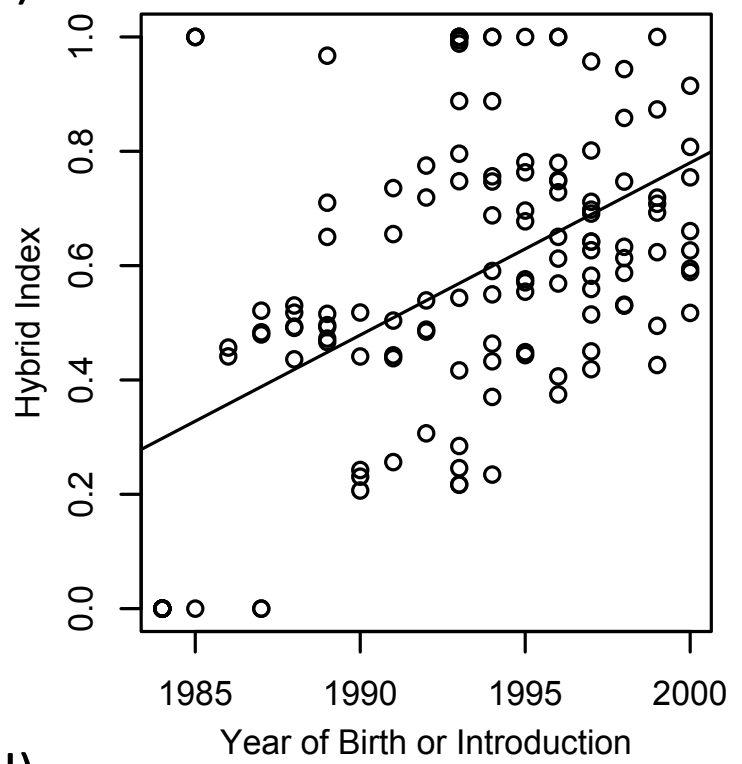

(d)

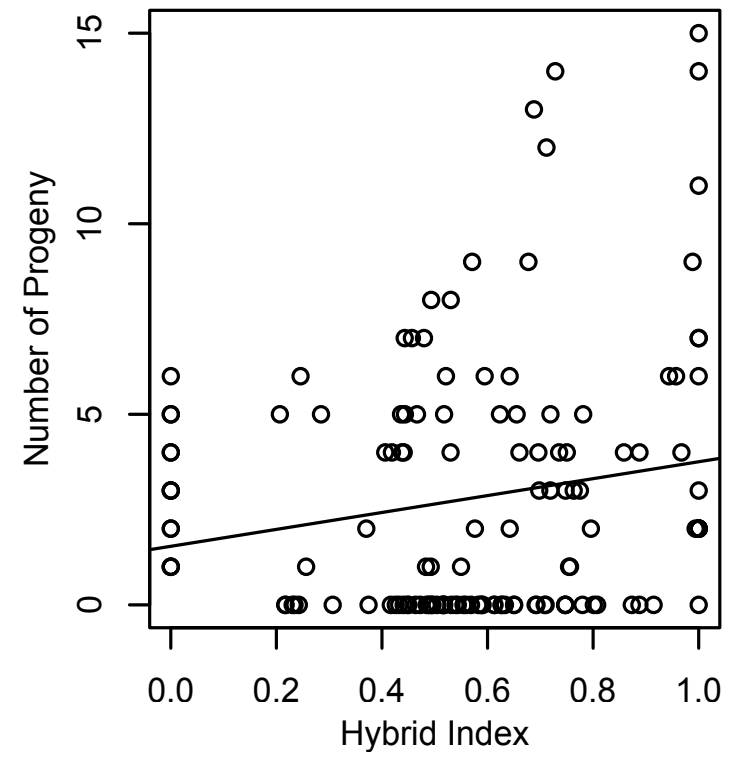


Conservation issues management

Scientific consulting (with genomics expertise)

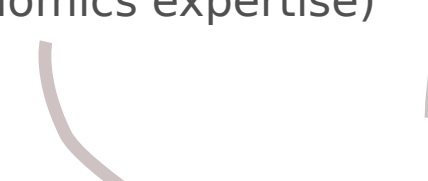

\section{Real-world Fundamental

$$
\text { research }
$$ research evaluations \\ Publications and}

Academia

Grant funding

Impact review

conservation issues

Uptake or

application 
Figure in Box 3

Genomics research and development

SNP discovery

SNP validation and selection

Genome-wide genotyping

Marker assessment and selection

Population screening

Population genomic analysis

SNP panel selection

Applied traceability tools

Platform selection

Method validation

Standard Operating Procedures

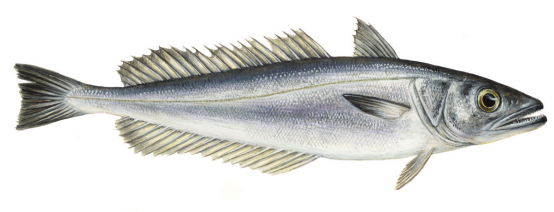

European hake (Merluccius merluccius L.)

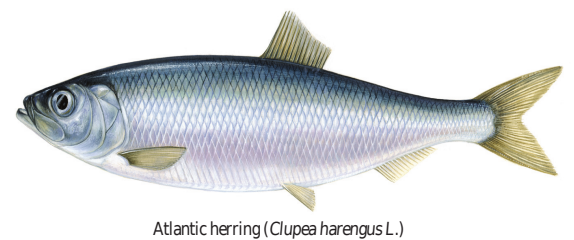

Atlantic herring (Clupea harengus L.)
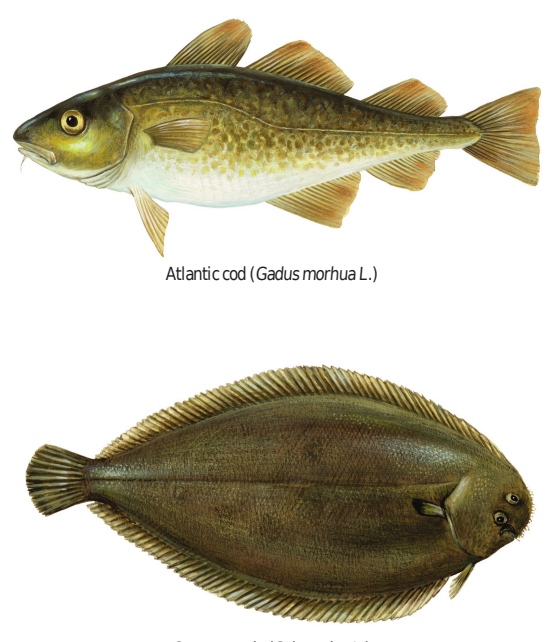

Common sole (Solea solea L.) 